\title{
The Hydration Repulsion between Charged Surfaces as an Interplay of Volume Exclusion and Dielectric Saturation Effects
}

\author{
V. N. Paunov, $*$ R. I. Dimova, $*$ P. A. Kralchevsky, ${ }^{* 1}$ G. Broze, $\dagger$ and A. Mehreteab $\ddagger$ \\ * Laboratory of Thermodynamics and Physico-chemical Hydrodynamics, Faculty of Chemistry, University of Sofia, 1126 Sofia, Bulgaria; \\ $\dagger$ Colgate-Palmolive R \& D, Inc., Avenue Du Parc Industriel, B-4041 Milmort, Belgium; and $\ddagger$ Colgate-Palmolive Co., \\ Technology Center, 909 River Road, Piscataway, New Jersey 08854-5596
}

Received December 19, 1995; accepted March 13, 1996

\begin{abstract}
We demonstrate that the hydration repulsion between smooth charged surfaces can be attributed to the interplay of two effects which are not taken into account in the conventional DLVO theory. These are the finite size of the counterions and the variable dielectric permittivity across the electric double layers. W e involve these two effects in the theory by coupling the Poisson - B oltzmann equation with the Bikerman and Booth equations. The resulting nonlinear ordinary differential equation of second order is solved numerically. The theory is applied to interpret available experimental data for the dependence of the surface force on the distance measured by means of the surface force apparatus. Excellent agreement between theory and experiment is obtained with reasonable values of the two adjustable parameters: the area per surface charge and the counterion adsorption energy. It turns out that the contribution of the ionic-excluded volume to the hydration force is several times greater than the contribution due to the dielectric saturation. The developed theoretical approach can find application for interpreting data about the interactions in aqueous thin films, colloidal dispersions, and micellar surfactant solutions in the presence of electrolyte at ionic strengths above $1 \mathrm{mM}$. $\odot 1996$ Academic Press, Inc.

Key Words: counterion adsorption; dielectric saturation; hydration energy of ions; hydration repulsion; surface force apparatus; volume exclusion effect for ions.
\end{abstract}

\section{INTRODUCTION}

In their review on hydration forces Israelachvili and Wennerström (1) note that hydration forces are now routinely invoked to explain any unexpected repulsion and/or swelling of colloids, clays, soap films, and amphiphilic structures in water. It turns out that effects of different physical origin are termed "hydration forces" in the literature. That is the reason why from the very beginning we specify that in the present study we deal with the short-range monotonic hydration repulsion which appears between two molecularly smooth charged surfaces across an electrolyte solution. Such forces were measured

\footnotetext{
${ }^{1}$ To whom correspondence should be addressed.
}

by Israelachvili et al. $(2,3)$ and Pashley $(4,5)$, who examined the validity of DLVO theory at small film thicknesses in experiments with films from aqueous electrolyte solutions confined between two mica surfaces. At electrolyte concentrations below $10^{-4} M\left(\mathrm{KNO}_{3}\right.$ or $\left.\mathrm{KCl}\right)$ they observed the typical DLVO maximum. However, at electrolyte concentrations higher than $10^{-3} M$ they did not observe the expected DLVO maximum and primary minimum; instead a strong short-range repulsion was detected, see also Ref. (6). Empirically, this force called the "hydration repulsion" appears to follow an exponential law (6)

$$
f_{\text {hydr }}(h)=f_{0} e^{-h / \lambda_{0}},
$$

where the decay length $\lambda_{0} \approx 0.6-1.1 \mathrm{~nm}$ for $1: 1$ electrolytes and $f_{0}$ depends on the hydration of the surfaces but is usually in the range of $3-30 \mathrm{~mJ} / \mathrm{m}^{2}$.

The physical importance of the hydration force is that it stabilizes some dispersions preventing coagulation in the primary DLVO minimum. It is believed that the hydration force is connected with the binding of strongly hydrated ions at the interface. This is probably the explanation of the experimental results of Healy et al. (7), who found that even high electrolyte concentrations cannot cause coagulation of amphoteric latex particles due to binding of strongly hydrated $\mathrm{Li}^{+}$ions at the particle surfaces. If the $\mathrm{Li}^{+}$ions are replaced by weakly hydrated $\mathrm{Cs}^{+}$ions, the hydration repulsion becomes negligible compared with the van der Waals attraction and the particles coagulate as predicted by the DLVO theory.

For the time being there is no generally accepted theory of the repulsive hydration forces. The first quantitative theory by Marčelja and Radič ( 8 ) attributes the hydration repulsion to the water structuring in a vicinity of a surface, which leads to the appearance of a decaying polarization profile. This model was further developed by other authors $(9,10)$. A different approach was proposed by Jönsson and Wennerström (11), who developed an explicit electrostatic model based on the image charge concept. Leikin and Kornyshev 
(12) combined the main features of the solvent polarization (8) and image charge (11) models in a nonlocal electrostatic theory of the repulsion between electroneutral lipid bilayers. On the other hand, Israelachvili and Wennerström (1) demonstrated that the short-range repulsion between lipid membranes may also be a manifestation of undulation, peristaltic, and protrusion forces, which are due to thermally excited fluctuations at the interfaces.

In the case of charged surfaces, which are the subject of the present work, Henderson and Losada-Cassou $(13,14)$ pointed out that the physical origin of the hydration repulsion can be attributed to the presence of a layer of lower dielectric constant, $\varepsilon$, in a vicinity of the interface. These authors considered the effects of the finite size of both ions and solvent molecules in the framework of the Percus-Yevick closure for hard spheres. It was demonstrated that the DLVO theory complemented with such a layer predicts correctly the dependence of hydration repulsion on the electrolyte concentration (15). A further extension of this approach was given by Basu and Sharma (16), who incorporated the effect of the variation of $\varepsilon$ in the theory of electrostatic disjoining pressure. Their model provides quantitative agreement with the experimental data at low electrolyte concentration and $\mathrm{pH}$, and qualitative agreement at higher electrolyte concentration and $\mathrm{pH}$.

The aim of the present article is to demonstrate that if the theory of Basu and Sharma (16) is further extended by taking into account the finite size of the ions, then quantitative agreement between theory and experiment can be achieved even for comparatively high electrolyte concentrations and $\mathrm{pH}$.

In the next section we review the basic equations for the electric potential in a thin film, accounting also for the effects of the ionic-excluded volume and the dielectric saturation. Further we consider the expressions for calculating the hydration repulsion contribution to the disjoining pressure and surface free energy. Next we compare the theoretical predictions with available experimental data $(4,5,17,18)$ for the hydration force measured by the surface force apparatus. Finally we discuss the dependence of the hydration repulsion on the electrolyte concentration and the relative contributions of the effects of volume exclusion and dielectric saturation.

\section{BASIC EQUATIONS}

We consider the case of planar geometry. The Poisson equation has the form

$$
\frac{d}{d z}\left(\varepsilon \frac{d \psi}{d z}\right)=-4 \pi \rho(z)
$$

where the $z$ axis is perpendicular to the interface $(s), \psi(z)$ is the electric potential, and $\rho(z)$ is the electric charge density.

\section{a. Effect of the Dielectric Saturation}

In general, the dielectric permittivity, $\varepsilon$, is a function of the electric field strength, $E=-d \psi / d x$. Following Gur et al. (19) and Basu and Sharma (16) we will use the expression for $\varepsilon(E)$ taken from Booth (20)

$$
\varepsilon(E)=n^{2}+\left(\varepsilon_{\mathrm{b}}-n^{2}\right) \frac{3}{\beta E}\left(\operatorname{coth} \beta E-\frac{1}{\beta E}\right)
$$

where $n=1.33$ is the refraction index of water, $\varepsilon_{\mathrm{b}}$ is the bulk dielectric constant (for $E=0$ ),

$$
\beta \equiv \frac{5 \mu}{2 k T}\left(n^{2}+2\right),
$$

and $\mu=1.85 \times 10^{-18}$ (CGSE units) is the dipole moment of the water molecule; $k$ is the Boltzmann constant and $T$ is the temperature.

In our study we have neglected the effect due to the finite size of the solvent molecules, considering them as a dielectric continuum. This is a common assumption used with the Poisson-Boltzmann equation but here the effect of the solvent polarization is also taken into account through the Booth equation [2.2].

\section{b. Hydration Energy of the Ions}

Following Gur et al. (19) we express the excess free energy of hydration of an ion (of the $i$ th species) by means of the Born (21) formula

$$
W_{i}=\frac{Z_{i}^{2} e^{2}}{2 r_{i}}\left(\frac{1}{\varepsilon(E)}-\frac{1}{\varepsilon_{\mathrm{b}}}\right) \text {, }
$$

where $Z_{i}$ is the valence of the $i$ th ion and $r_{i}$ is its radius; $e$ is the elementary electric charge. Then the dimensionless potential energy of the $i$ th ion in the electric double layer can be expressed in the form (19)

$$
U_{i}=-\frac{Z_{i} e \psi+W_{i}}{k T}
$$

In fact, $U_{i}$ is to be substituted in the Boltzmann equation in order for the ionic distribution to be calculated, see below.

\section{c. Volume Exclusion Effect Due to the Ions}

Since the concentration of the counterions is high in the vicinity of a charged interface, the ionic volume exclusion effect becomes important. We take into account this effect by using the Bikerman equation, see Refs. (22) and (23): 


$$
n_{i}(z)=\frac{1-v \sum_{k} n_{k}(z)}{1-v \sum_{k} n_{k 0}} n_{i 0} \exp U_{i}
$$

Here $n_{i}$ is the number density of the $i$ th ion in the double layer, $n_{i 0}$ is the value of $n_{i}$ in the bulk solution where $U_{i}=$ 0 , the summation is carried out over all ionic species; $v$ has the meaning of average excluded volume per ion. It turns out that $v$ is about eight times the volume of the hydrated counterion. The derivation of Bikerman equation [2.6] is based on the approximate "free volume"' approach; see Appendix I where the meaning and the value of the parameter $v$ are also discussed. Equation [2.6] is expected to give correct results for not too high electrolyte concentrations. It is convenient to introduce the notation

$$
n_{i}^{*}=\frac{n_{i 0}}{1-v \Sigma_{k} n_{k 0}} .
$$

Using Eqs. [2.6] and [2.7] one can derive

$$
n_{i}=\frac{n_{i}^{*} \exp U_{i}}{1+v \Sigma_{k} n_{k}^{*} \exp U_{k}} .
$$

Further, one can express the charge density in the form

$$
\rho=\frac{\sum_{i} Z_{i} e n_{i}^{*} \exp U_{i}}{1+v \sum_{i} n_{i}^{*} \exp U_{i}}
$$

Note that $Z_{i}$ is defined with its algebraic sign: $Z_{i}$ is positive for cations and negative for anions.

\section{d. Boundary Conditions and Computational Procedure}

The set of Eqs. [2.1], [2.2], [2.5], and [2.9] allows one to determine the electrical potential, $\psi(z)$, and the distributions of the ionic species, $n_{i}(z)$. The inclusion of the effects of variable $\varepsilon$ and ionic volume in the theory makes impossible to find an explicit analytical solution. Therefore, we use numerical solution of the problem. It is interesting to note that the effect of finite ionic size improves the stability of the numerical procedure: some computational difficulties encountered when solving the Poisson-Boltzmann equation for point ions do not appear. The latter difficulties are related to the unrealistically high subsurface ionic concentration obtained often for point ions. Moreover, the solution is always unique; we do not obtain double solution for the film midpoint potential as reported in Ref. (16) for point ions. The procedure of calculation we used is the following.

After Gur et al. (19) we introduce the dimensionless variables

$$
x \equiv \exp (-\kappa z), \quad \varphi \equiv e \psi /(k T),
$$

where

$$
\kappa=\left[\frac{4 \pi e^{2}}{\varepsilon k T} \sum_{i} Z_{i}^{2} n_{i 0}\right]^{1 / 2}
$$

is the reverse Debye screening length. The introduction of the variable $x$ provides an appropriate mapping of the subsurface region $(z \rightarrow 0)$, where the potential $\varphi(z)$ exhibits fast variation; this allows one to use an equidistant set of nodes with respect to $x$ when solving the problem numerically.

By using Eq. [2.10] one can represent the Poisson equation, Eq. [2.1], in the form

$$
\frac{d^{2} \varphi}{d x^{2}}=f\left(x, \varphi, \varphi^{\prime}\right), \quad \varphi^{\prime} \equiv \frac{d \varphi}{d x}
$$

where

$f\left(x, \varphi, \varphi^{\prime}\right)=-\frac{4 \pi e \rho}{\varepsilon+E(d \varepsilon / d E)} \frac{1}{k T \kappa^{2} x^{2}}-\frac{1}{x} \varphi^{\prime}$

and

$$
E=k T \kappa x \varphi^{\prime} / e
$$

The boundary condition on the midplane of the film reads

$$
\left.\varphi^{\prime}\right|_{x=x_{\mathrm{m}}}=0
$$

where $x_{\mathrm{m}}$ denotes the value of $x$ in the midplane of the film. The boundary condition on the film surface can be imposed in three alternative ways:

(i) regime of constant surface potential, $\varphi_{\mathrm{s}}$ :

$$
\left.\varphi\right|_{x=1}=\varphi_{\mathrm{s}}=\mathrm{const}
$$

(ii) regime of constant surface charge density, $\sigma_{\mathrm{s}}$

$$
\left.\frac{d \varphi}{d x}\right|_{x=1}=\frac{4 \pi e \sigma_{\mathrm{s}}}{\kappa \varepsilon_{\mathrm{s}} k T}=\mathrm{const} \quad\left(\left.\varepsilon_{\mathrm{s}} \equiv \varepsilon\right|_{x=1}\right) ;
$$

(iii) regime of surface charge regulation (see e.g., Ref. (24))

$$
\left.\frac{d \varphi}{d x}\right|_{x=1}=\frac{4 \pi e^{2} Z_{\mathrm{s}} \Gamma_{\mathrm{s}}}{\varepsilon k T \kappa\left(1+\left(n_{1 \mathrm{~s}} \delta_{1} / \Gamma_{\mathrm{s}}\right) \exp (\Phi / k T)\right)},
$$

where $\delta_{1}$ is the diameter of the counterions, $n_{1 \mathrm{~s}}$ is their subsurface concentration, 


$$
n_{1 \mathrm{~s}}=\left.\frac{n_{1}^{*} \exp U_{1}}{1+v \sum_{k} n_{k}^{*} \exp U_{k}}\right|_{x=1}
$$

cf. Eq. [2.8]. To obtain Eq. [2.18] we used the Stern isotherm; $\Gamma_{\mathrm{s}}$ is the number density of the surface ionizable groups, which play the role of adsorption centers for the counterions, and $Z_{\mathrm{s}}\left(Z_{\mathrm{s}}= \pm 1\right)$ denotes the sign of the surface charge. The solution of the problem gives $\varphi=\varphi(x)$ and, in particular, the value $\varphi_{\mathrm{m}} \equiv \varphi\left(x_{\mathrm{m}}\right)$ in the midplane of the film. The numerical method is described in Appendix II.

\section{ELECTROSTATIC AND HYDRATION SURFACE FORCES}

After Verwey and Overbeek (25), and Basu and Sharma (16) we use the expression

$$
\Pi_{\mathrm{el}}=-\int_{0}^{\psi_{\mathrm{m}}} \rho_{\mathrm{m}}\left(\psi_{\mathrm{m}}\right) d \psi_{\mathrm{m}}=-\frac{k T}{e} \int_{0}^{\varphi_{\mathrm{m}}} \rho_{\mathrm{m}}\left(\varphi_{\mathrm{m}}\right) d \varphi
$$

to calculate the electrostatic component of disjoining pressure, $\Pi_{\mathrm{el}}$; here $\rho_{\mathrm{m}}$ is the value of the charge density, $\rho$, in the midplane of the film.

Since $E=0$ in the midplane, $\rho_{\mathrm{m}}$ is defined by Eq. [2.9] with $U_{i}=-Z_{i} \varphi_{\mathrm{m}}$. Then the integration in Eq. [3.1] can be carried out to give

$$
\Pi_{\mathrm{el}}=\frac{k T}{v} \ln \left[\frac{1+v \Sigma_{k} n_{k}^{*} \exp \left(-Z_{k} \varphi_{\mathrm{m}}\right)}{1+v \sum_{k} n_{k}^{*}}\right] .
$$

The ionic volume exclusion effect enters Eq. [3.2] both explicitly (through $v$ and $n_{k}^{*}$, cf. Eq. [2.7]) and implicitly (through $\varphi_{\mathrm{m}}$, which is calculated by means of the procedure described in the previous section). The effect of the variable dielectric constant $\varepsilon(E)$ affects Eq. [3.2] only implicitly, through $\varphi_{\mathrm{m}}$. Note also that $\varphi_{\mathrm{m}}$ depends on the film thickness, $h$, and consequently, Eq. [3.2] determines the function $\Pi_{\mathrm{el}}(h)$. The excess free energy per unit area due to the electrostatic interactions across the film is (26)

$$
f_{\mathrm{el}}=\int_{h}^{\infty} \Pi_{\mathrm{el}}(h) d h
$$

For $\kappa h \gg 1$ the effects of both volume exclusion and variable $\varepsilon$ become negligible and $\Pi_{\mathrm{el}}$ decays exponentially,

$$
\Pi_{\mathrm{el}}(h)=\Pi_{\mathrm{el}}\left(h_{N}\right) e^{-\kappa\left(h-h_{N}\right)}, \quad h \geqslant h_{N}\left(\kappa h_{N} \gg 1\right)
$$

The latter asymptotic expression can be utilized to make the integration domain in Eq. [3.3] finite:

$$
f_{\mathrm{el}}(h)=\int_{h}^{h_{N}} \Pi_{\mathrm{el}}(h) d h+\frac{1}{\kappa} \Pi_{\mathrm{el}}\left(h_{N}\right) e^{-\kappa\left(h-h_{N}\right)} .
$$

The disjoining pressure due to hydration repulsion can be defined as

$$
\Pi_{\mathrm{hr}}(h)=\Pi_{\mathrm{el}}(h)-\Pi_{\mathrm{el}}^{(0)}(h)
$$

where $\Pi_{\mathrm{el}}^{(0)}(h)$ is the electrostatic disjoining pressure provided by the conventional DLVO theory. To calculate $\Pi_{\mathrm{el}}^{(0)}$ one may use the Langmuir formula (27)

$$
\Pi_{\mathrm{el}}^{(0)}=k T\left[\sum_{k} n_{k 0} \exp \left(-Z_{k} \varphi_{m}^{(0)}\right)-\sum_{k} n_{k 0}\right],
$$

which follows from Eqs. [2.7] and [3.2] for $v \rightarrow 0$ and $\varphi_{\mathrm{m}} \rightarrow \varphi_{\mathrm{m}}^{(0)}$. Here $\varphi_{\mathrm{m}}^{(0)}$ is the potential in the midplane calculated with $v=0$ and $\varepsilon=\varepsilon_{\mathrm{b}}$. Note that the effects of both ionic size and variable $\varepsilon$ lead to $\left|\varphi_{\mathrm{m}}\right|>\left|\varphi_{\mathrm{m}}^{(0)}\right|$. This contributes to a positive $\Pi_{\mathrm{hr}}$ in Eq. [3.6].

\section{NUMERICAL RESULTS AND DISCUSSION}

The hydration repulsion between two crossed cylinders of radius $R$ was measured across electrolyte solutions by means of the surface force apparatus $(1-5,17,18)$. The experiment gives the quantity $F / R$, where $F$ denotes force. On the other hand, the Derjaguin approximation (26) yields $F / R=2 \pi f$, where $f=f(h)$ is the surface excess free energy. Theoretically, $f$ can be expressed in the form

$$
f(h)=f_{\mathrm{el}}(h)+f_{v w}(h), \quad f_{v w}(h)=-\frac{A_{H}}{12 \pi h^{2}},
$$

where $f_{\mathrm{el}}$ is given by Eq. [3.5]; we use the value $A_{\mathrm{H}}=2.2$ $\times 10^{-20} \mathrm{~J}$ for the compound Hamaker constant of an aqueous film between two mica surfaces (6). In view of Eqs. [3.5] and [3.6], $f_{\mathrm{el}}(h)$ accounts for both hydration and DLVO electrostatic repulsion. Note that the effect of the finite size of the solvent molecules leads to oscillations superimposed over the monotonic hydration repulsion. These oscillations are often considered as a separate oscillatory structural surface force $(6,29)$, which is not a subject of the present study. Below we compare available experimental data with the predictions of the theory presented in Sections 2 and 3 above. As boundary condition at the film surfaces we use the condition for charge regulation, Eq. [2.18].

The theory contains three parameters: $v, a \equiv 1 / \Gamma_{\mathrm{s}}$, and $\Phi$, see Eqs. [2.6] and [2.18]. On the basis of the analysis given in Appendix I we fix the excluded volume $v$ to be equal to 8 times the volume of a hydrated counterion; we used the value $0.33 \mathrm{~nm}$ for the radius of $\mathrm{K}^{+}$ion and 0.36 for the radius of $\mathrm{Na}^{+}$ion (6). The other two parameters, viz. 


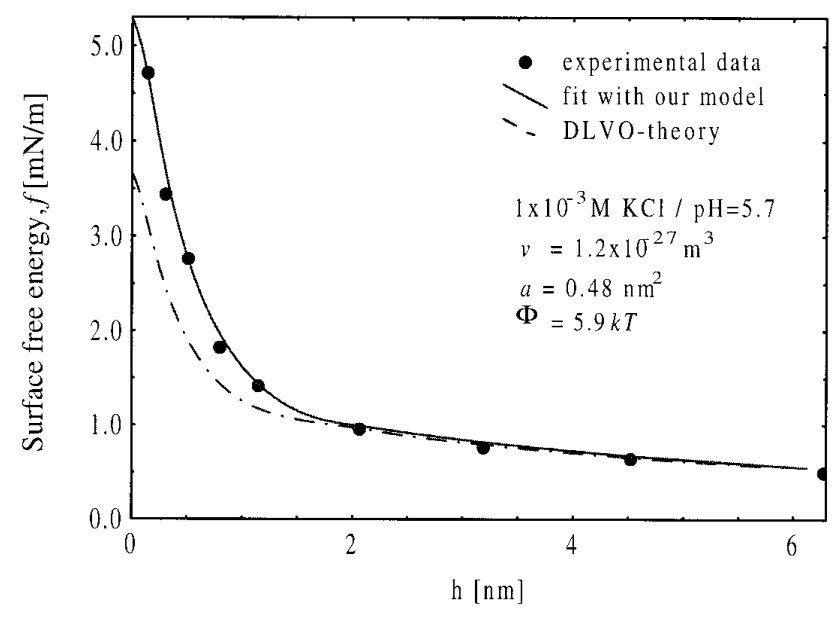

FIG. 1. Plot of surface free energy, $f$, vs film thickness, $h$. The points are experimental data from Fig. 3 in Ref. (5) measured with solution of 1 $\times 10^{-3} \mathrm{KCl} M$ at $\mathrm{pH} 5.7$ between mica surfaces. The full line is the best fit corresponding to $\Phi=5.9 \mathrm{kT}$. The area per surface charge is fixed to $a$ $=0.48 \mathrm{~nm}^{2}$

the area per surface charge, $a$, and the energy of adsorption (binding) per counterion, $\Phi$, are treated as adjustable parameters; we determine the value of $a$ and $\Phi$ from the best fit of the data by the least-squares method. In the case of mica the value of $a$ is known, and in this specific case we treat only $\Phi$ as an adjustable parameter (see below).

Figures 1-5 represent the comparison between our theory (the solid curves) with the experimental data of other authors for the surface force. The dashed curves give the predictions of the conventional DLVO theory (26) for the same values of the parameters.

In Fig. 1 we compare the theory with the experimental data by Pashley (5) for $1 \times 10^{-3} \mathrm{M} \mathrm{KCl}$ solution at $\mathrm{pH}$ 5.7. The film is formed between two molecularly smooth mica surfaces. Since the area per surface ionizable group for mica is known (see e.g., Ref. (30)) we fixed $a=0.48 \mathrm{~nm}^{2}$ and the data were fitted by varying only the adsorption energy, $\Phi$, of the $\mathrm{K}^{+}$ions. Excellent agreement between theory and experiment is observed. From the best fit we determine the energy of adsorption of a $\mathrm{K}^{+}$ion to be $\Phi=5.9 \mathrm{kT}$.

In Fig. 2 we present a similar plot, but for higher electrolyte concentration: $2.3 \times 10^{-3} \mathrm{M} \mathrm{KCl} / \mathrm{KOH}$ at $\mathrm{pH} 11.1$. The data is taken from Ref. (4). The area per surface ionizable group is again $a=0.48 \mathrm{~nm}^{2}$; the calculated value of the adsorption energy of a $\mathrm{K}^{+}$ion, $\Phi=5.6 \mathrm{kT}$, is almost the same as in Fig. 1.

The fact that the best fit gives close values of the adsorption energy $\Phi$ per $\mathrm{K}^{+}$ion for two different sets of data, Figs. 1 and 2, is an argument in favor of the physical adequacy of our theoretical model.

Figure 3 shows data of Pashley (4) for $\mathrm{Na}^{+}$counterions (instead of $\mathrm{K}^{+}$). The film is formed again between mica surfaces. The full line in Fig. 3 represents the best fit corresponding to adsorption energy per $\mathrm{Na}^{+}$ion $\Phi=3.5 \mathrm{kT}$.

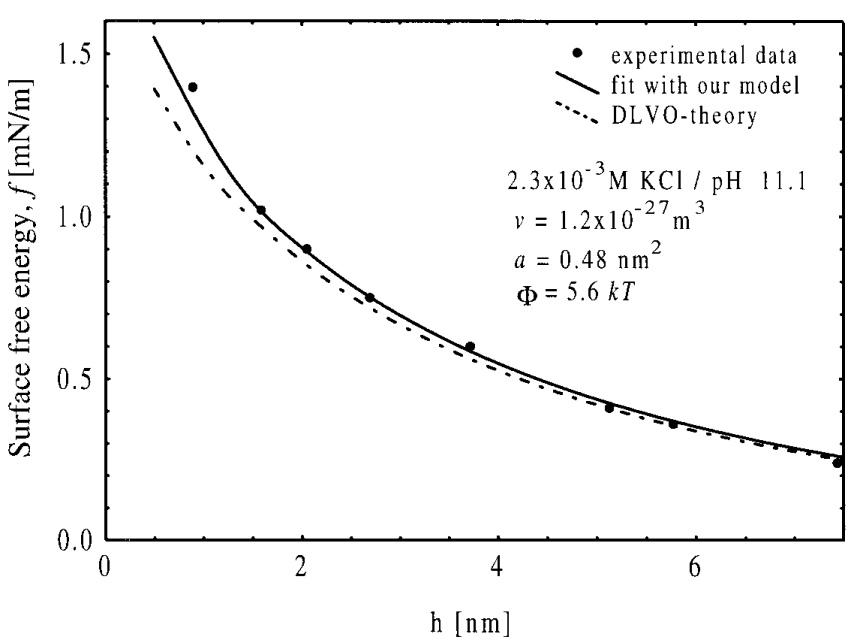

FIG. 2. Plot of $f$ vs $h$. The points are experimental data from Fig. 8 in Ref. (4) measured with solution of $2.3 \times 10^{-3} \mathrm{M} \mathrm{KCl} / \mathrm{KOH}$ at $\mathrm{pH} 11.1$ between mica surfaces. The full line is the best fit corresponding to $\Phi=$ $5.6 \mathrm{kT}$. The area per surface charge is fixed to $a=0.48 \mathrm{~nm}^{2}$.

Figure 4 also shows data for $\mathrm{Na}^{+}$counterions (taken from Horn et al., Ref. (17)), but this time the salt concentration is higher, $0.11 \mathrm{M} \mathrm{NaCl}$, and the film is formed between silica (instead of mica) sheets. The data are more scattered as compared with Figs. 1-3, but nevertheless there is a good agreement between theory and experiment. Since the electrolyte concentration is higher, one may expect that some effect of the ionic correlation surface force could appear. For that reason we employed the theory in Ref. (28) to calculate the contribution of the ionic correlations. As seen in Fig. 4 the curves calculated with and without accounting for the ionic correlations almost coincide; i.e., the effect of the ionic correlations is negligible even for this relatively high salt con-

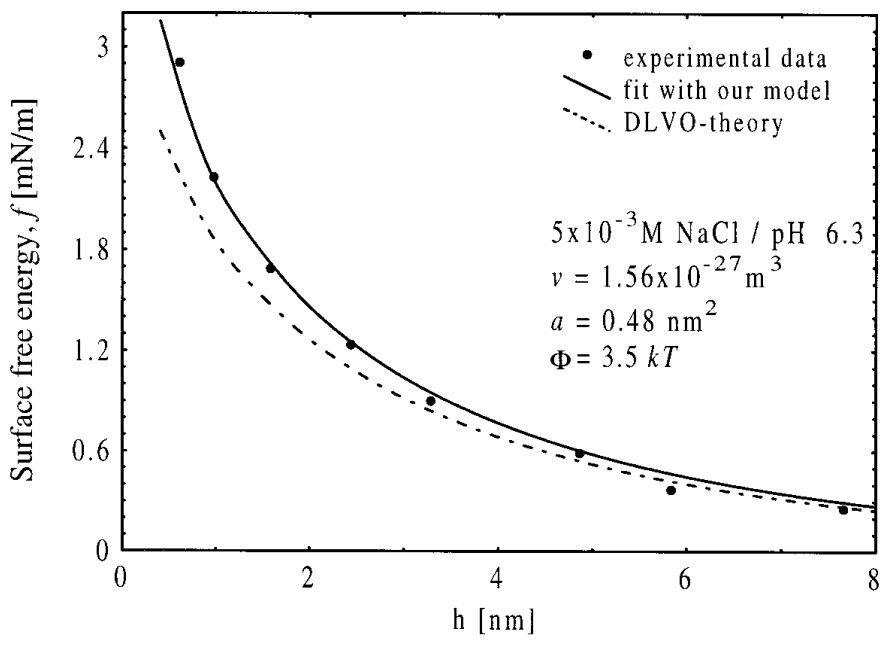

FIG. 3. Plot of $f$ vs $h$. The points are experimental data from Fig. 4 in Ref. (4) measured with solution of $5 \times 10^{-3} \mathrm{M} \mathrm{NaCl}$ at $\mathrm{pH} 6.3$ between mica surfaces. The line is the best fit corresponding to $\Phi=3.5 k T$. The area per surface charge is fixed to $a=0.48 \mathrm{~nm}^{2}$. 


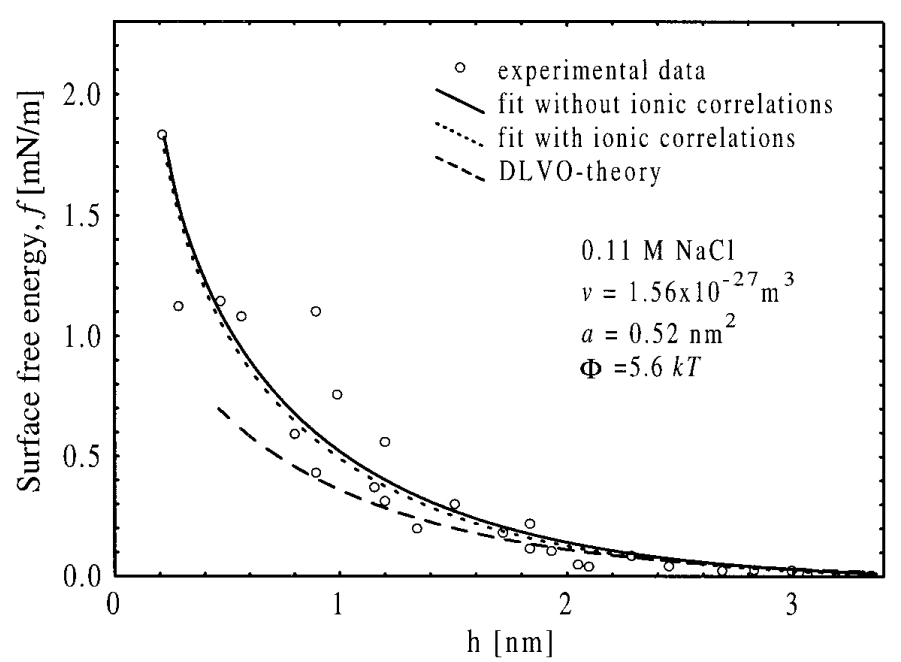

FIG. 4. Plot of $f$ vs $h$. The points are experimental data from Fig. 2 in Ref. (17) measured with solution of $0.11 \mathrm{M} \mathrm{NaCl}$ at $\mathrm{pH} 5.7$ between silica surfaces. The full line is the best fit corresponding to $a=0.52 \mathrm{~nm}^{2}$ and $\Phi=5.6 \mathrm{kT}$; the short-dashed line accounts for the contribution of the ionic correlation surface forces.

centration (this would not be the case if a $2: 2$ electrolyte was present, see Ref. (28)). Since the surface structure is not well defined and depends on the prehistory of the silica sheets (formation of gel layer of hydrolyzed silica acid), we use both $a$ and $\Phi$ as adjustable parameters. The calculated area per surface charge of the silica sheet, $a=0.52 \mathrm{~nm}^{2}$, turns out to be larger than the value of $a$ for mica sheets, cf. Figs. 1-3. In addition, the calculated adsorption energy of a $\mathrm{Na}^{+}$ion on silica, $\Phi=5.6 \mathrm{kT}$, is somewhat larger compared to the calculated value for mica $(\Phi=3.5 k T$, cf. Fig. 3).

Figure 5 presents similar data, but for another type of film

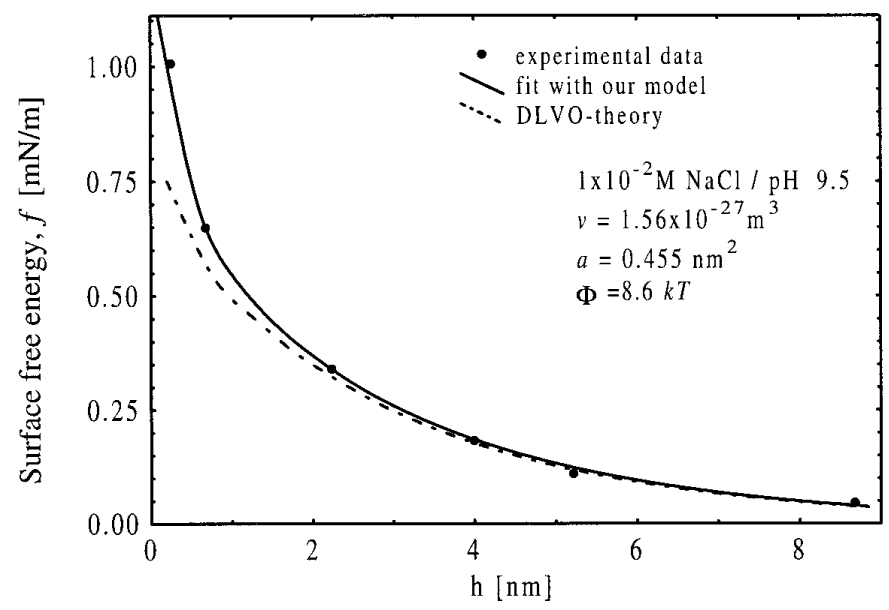

FIG. 5. Plot of $f$ vs $h$. The points are experimental data from Fig. 1 in Ref. (18) measured with solution of $1 \times 10^{-2} \mathrm{M} \mathrm{NaCl}$ at $\mathrm{pH} 5.7$ between two deposited monolayers of dihexadecyl phosphate. The full line is the best fit corresponding to $a=0.455 \mathrm{~nm}^{2}$ and $\Phi=8.6 \mathrm{kT}$.

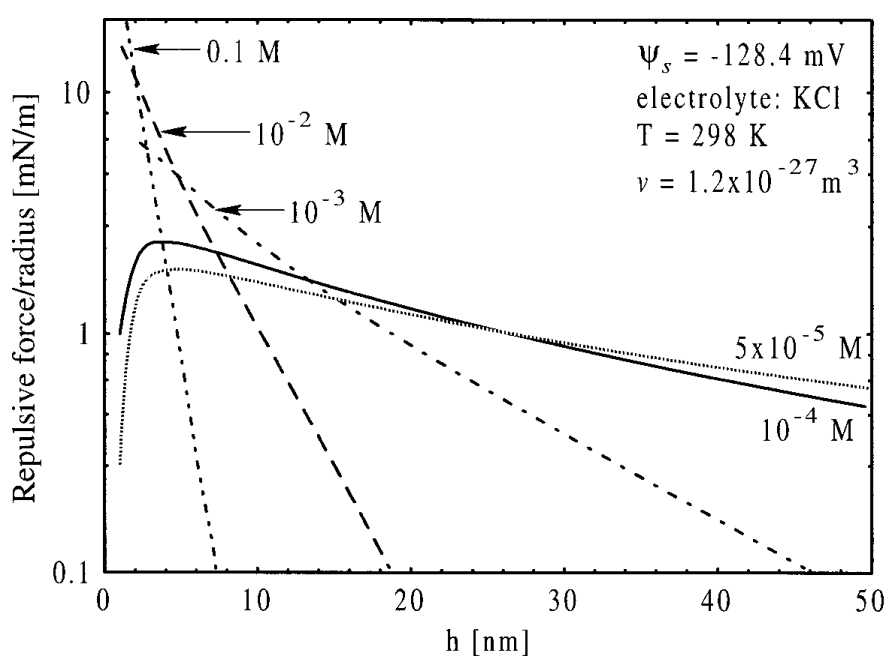

FIG. 6. Theoretical plot of $F / R=2 \pi f$ vs $h$ for various concentrations of $\mathrm{KCl}$ denoted on the curves. All curves are drawn for the same values of the surface potential $\psi_{s}=-128.4 \mathrm{mV}$, excluded volume of $\mathrm{K}^{+}$ion $v=$ $1.2 \times 10^{-27} \mathrm{~m}^{3}$ and $T=298 \mathrm{~K}$.

surfaces: deposited monolayers of dihexadecyl phosphate (DHP). The data are taken from Claesson et al. (18). The solution contains $1 \times 10^{-2} \mathrm{M} \mathrm{NaCl}$ at $\mathrm{pH} 9.5$. At this value of $\mathrm{pH}$ one may expect that DHP (initially in acidic form) is highly dissociated. This expectation is consonant with the relatively small area per surface charge, $a=0.455 \mathrm{~nm}^{2}$, obtained from the best fit. In addition, the calculated higher value of the adsorption energy per $\mathrm{Na}^{+}$ion, $\Phi=8.6 \mathrm{kT}$, evidences that the $\mathrm{Na}^{+}$ions bind stronger to the DHP layers than to the mica or silica sheets, cf. Figs. 3 and 4.

In Fig. 6 we investigate the dependence of hydration repulsion on the concentration of electrolyte $(\mathrm{KCl})$. All theoretical curves are calculated for $v=1.2 \times 10^{-27} \mathrm{~m}^{3}, A_{H}=2.2 \times$ $10^{-20} \mathrm{~J}$, and $\psi_{\mathrm{s}}=-128.4 \mathrm{mV}$; the boundary conditions for constant potential, Eq. [2.16], was used. We plot $F / R=2 \pi f$ vs $h$. The theoretical curves in Fig. 6 resemble very much the experimental findings, see, e.g., Fig. 13.9 in Ref. (6). In particular, for $C_{\mathrm{el}}=5 \times 10^{-5}$ and $10^{-4} \mathrm{M}$ a typical DLVO maximum is observed, without any indication about the existence of short-range repulsion. However, for $C_{\mathrm{el}}=10^{-3}, 10^{-2}$, and $10^{-1} M$ maximum is not seen but instead, the short-range hydration repulsion appears. Note that the increased electrolyte concentration increases the hydration repulsion, but suppresses the long-range double-layer repulsion, as it is known from previous studies. Note also that the experimentally observed oscillations superimposed on the monotonic $F / R$ vs $h$ curves can be reproduced if a term accounting for the oscillatory structural component of $f$ is added on the right-hand side of Eq. [4.1], see, e.g., Ref. (29).

In Fig. 7 we present theoretical plots of $\prod_{\mathrm{hr}}$ vs $h$ calculated by means of Eq. [3.6]. Our aim is to examine the effect of the ionic size on the hydration repulsion. For that reason we vary the excluded volume $v$ (which as before is taken to be 
equal to 8 times the volume of the counterion, see Appendix I) at fixed values $a=0.8 \mathrm{~nm}^{2}, \Phi=6 \mathrm{kT}$, and ionic strength $\mathrm{I}=0.01 M$. To calculate $v$ we used the values $0.33,0.38$, and $0.43 \mathrm{~nm}$ for the ionic radii of the hydrated $\mathrm{K}^{+}, \mathrm{Li}^{+}$, and $\mathrm{Mg}^{2+}$, respectively (6). One observes a marked increase of $\Pi_{\mathrm{hr}}$ with the increase of the counterion size. The lower curve is calculated with $v=0$; i.e., for this curve the value of $\Pi_{\mathrm{hr}}$ is determined only by the effect of dielectric saturation. This fact explains why we obtained an excellent agreement between theory and experiment despite the approximate character of Booth equation, Eq. [2.2], accounting for the effect of dielectric saturation. Indeed, the Booth equation is not accurate enough for small field strengths (19). However, the small relative contribution of the dielectric saturation to $\Pi_{\mathrm{hr}}$ (Fig. 7) implies that the correction of the Booth equation for small field strengths (16) is not so essential for the calculation of hydration repulsion.

The contour-plot diagram in Fig. 8 illustrates the range of electrolyte concentrations (1:1, electrolyte) and film thicknesses for which the effect of hydration repulsion is important. The values of the parameters are $v=1.2 \times 10^{-27}$ $\mathrm{m}^{3}, a=0.46 \mathrm{~nm}^{2}$, and $\Phi=4.8 k T$. Note that the larger the electrolyte concentration, the larger the effect of hydration repulsion.

It should be noted that in case of low $\mathrm{pH}$ the hydrated ions can be competed by $\mathrm{H}^{+}$ions for the adsorption sites at the film surfaces (see, e.g., Ref. (30)). Then, the boundary condition, Eq. [2.18], should be extended to account for the competitive adsorption of $\mathrm{H}^{+}$and the hydrated metal ion. However, it is observed experimentally (6) that in this case the effect of hydration repulsion disappears which can be attributed to the decrease in the subsurface concentration of the hydrated metal ions.

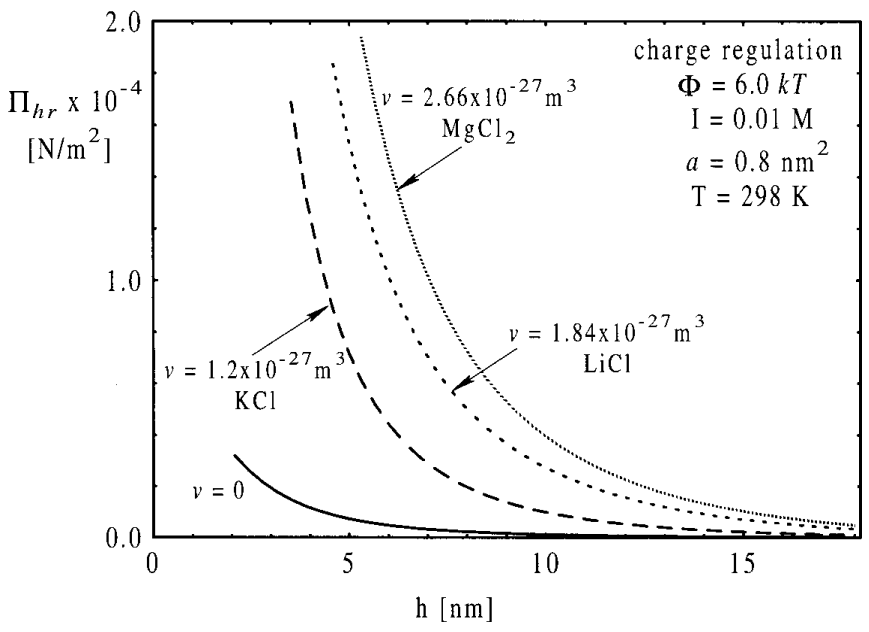

FIG. 7. Theoretical dependence of the hydration component of disjoining pressure, $\Pi_{\mathrm{hr}}$, on the film thickness, $h$, for various values of the excluded volume $v$; we use the values $v=2.66 \times 10^{-27} \mathrm{~m}^{3}$ for $\mathrm{Mg}^{2+}, v$ $=1.84 \times 10^{-27} \mathrm{~m}^{3}$ for $\mathrm{Li}^{+}$, and $v=1.2 \times 10^{-27} \mathrm{~m}^{3}$ for $\mathrm{K}^{+}$; the lower curve is calculated with $v=0$.

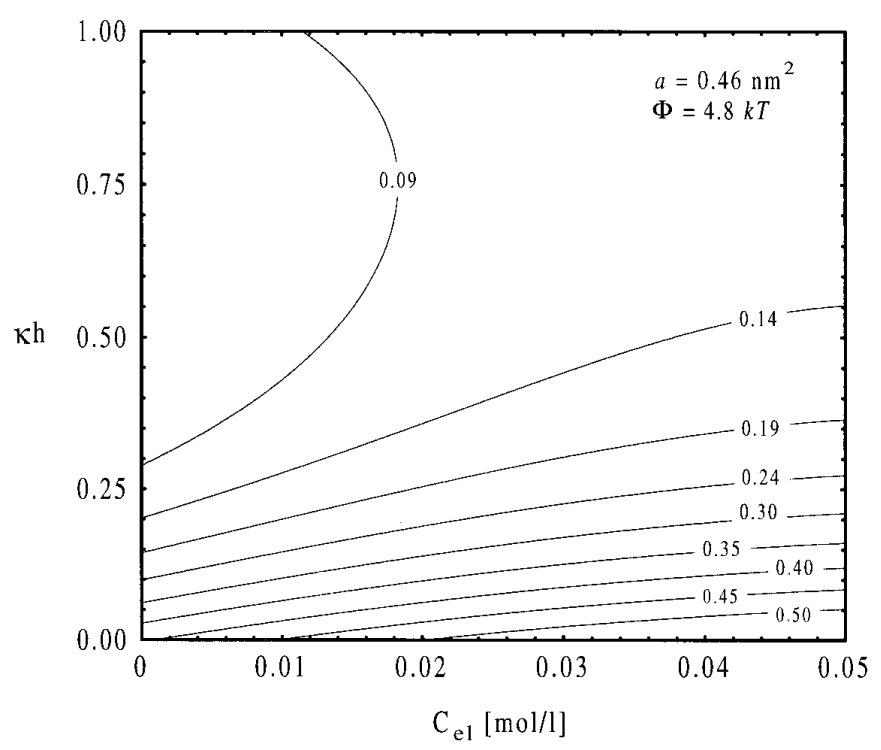

FIG. 8. Contour-plot diagram of the relative effect of hydration repulsion, $\Pi_{\mathrm{hr}} / \Pi_{\mathrm{el}}^{\mathrm{DLO}}$, vs the electrolyte concentration, $c_{\mathrm{el}}$, and the dimensionless film thickness, $\kappa h$. The values of the parameters are $v=1.2 \times 10^{-27} \mathrm{~m}^{3}$, $a=0.46 \mathrm{~nm}^{2}$, and $\Phi=4.8 \mathrm{kT}$. The contours correspond to fixed values of $\Pi_{\mathrm{hr}} / \Pi_{\mathrm{el}}^{\mathrm{DLVO}}$ denoted in the figure.

\section{CONCLUDING REMARKS}

In the present study we demonstrate that the hydration repulsion between smooth charged surfaces can be attributed to the interplay of two effects (not taken into account in the conventional DLVO theory): (i) the finite size of the counterions and (ii) variable dielectric permittivity.

The finite size of the counterions (the volume exclusion effect) is involved in the theory through the expression for the bulk charge density, Eq. [2.9], which is to be substituted in the Poisson equation, Eq. [2.1]. The volume exclusion effect is characterized by the Bikerman parameter $v$, which is approximately equal to 8 times the volume of the hydrated counterion, see Appendix I.

The effect of the variable dielectric permittivity, $\varepsilon(E)$, called also the dielectric saturation effect, is accounted for in the same way as in the earlier works by Gur et al. (19) and Basu and Sharma (16), see Eq. [2.2]. Note that $\varepsilon(E)$ enters the theory through the Poisson equation, Eq. [2.1], as well as through the ionic hydration energy in the Boltzmann equation, Eqs. [2.4], [2.5], and [2.9].

The resulting nonlinear ordinary differential equation of the second order is solved numerically. Accounting for the volume exclusion effect $(v>0)$ the numerical procedure becomes more stable and faster, and moreover, double solutions like those obtained by Basu and Sharma (16) do not appear.

The numerical procedure yields the value of the electric potential in the midplane of the film, $\varphi_{\mathrm{m}}$, which is then used to calculate the total electrostatic disjoining pressure, $\Pi_{\mathrm{el}}$, 
by means of Eq. [3.2]. $\Pi_{\mathrm{el}}$ can be considered as a sum of DLVO and hydration contributions, cf. Eq. [3.6].

The theory is applied to interpret available experimental data $(4,5,17,18)$ for the surface free energy $f$ vs the film thickness $h$. Two adjustable parameters are used to fit the data: the area per surface charge, $a$, and the adsorption energy of a counterion, $\Phi$. In the case of mica the value of $a$ is known, and in this specific case we treat only $\Phi$ as an adjustable parameter. The theory provides an excellent fit with reasonable values of $a$ and $\Phi$, see Figs. 1-5. The predictions of the theory are consonant with the experimental finding that the hydration repulsion is significant only for the higher electrolyte concentrations, $C_{\mathrm{el}} \geqslant 1 \mathrm{~m} M$, see Fig. 6 . The numerical test of the theory (Fig. 7) shows that the contribution of the volume exclusion to the hydration force is several times greater than the contribution due to dielectric saturation.

The theoretical approach developed in this study can find application for interpreting experimental data for the interactions in aqueous thin films, colloidal dispersions, and micellar surfactant solutions in the presence of electrolyte at ionic strengths above $1 \mathrm{~m} M$.

\section{APPENDIX I}

\section{On the Derivation of the Bikerman Equation}

In statistical mechanics the volume exclusion effect can be taken into account by substituting the volume of the system, $V$, with the so called "free volume,"

$$
V_{f}=V-N b, \quad N=\sum_{i} N_{i},
$$

in the partition function, see, e.g., Ref. (31). Here $N_{i}$ is number density of molecules of the $i$ th species and $b$ is an effective average volume occupied by a molecule. In a multicomponent system $b$ depends on the composition. The latter dependence can be estimated in the following way.

The use of Eq. [A.1] leads to the following expression for the free energy of a system of interacting molecules

$$
F=F_{\mathrm{id}}-N k T \ln \left(1-\frac{N b}{V}\right)-\frac{N^{2} c}{V}
$$

where $F_{\text {id }}$ is the free energy of an ideal gas; $b$ and $c$ account for the hard core and long-range interactions, respectively, cf. Refs. (31) and (32). In the limit of low concentrations Eq. [A.2] reduces to

$$
F=F_{\text {id }}+\frac{N^{2}}{V}(b k T-c) .
$$

The last equation is to be compared with the virial expansion $(31,32)$

$$
F=F_{\text {id }}+\frac{k T}{V} \sum_{i, j} N_{i} N_{j} B_{i j}(T)+\cdots
$$

where $B_{i j}$ are the second virial coefficients accounting for the two-particle interactions,

$$
B_{i j}=B_{j i}=2 \pi \int_{0}^{\infty} d r r^{2}\left[1-\exp \left(-\frac{U_{i j}(r)}{k T}\right)\right],
$$

where $U_{i j}(r)$ is the pair interaction energy between particles of the $i$ th and $j$ th species. If $r_{i j}$ is the center-to-center distance at closest approach between two particles of the respective species, one can write

$B_{i j}=b_{i j}-\frac{c_{i j}}{k T}, \quad c_{i j} \equiv-2 \pi \int_{r_{i j}}^{\infty}\left(1-\exp \left(-\frac{U_{i j}}{k T}\right)\right) r^{2} d r$

$b_{i j} \equiv 2 \pi \int_{0}^{r_{i j}}\left(1-\exp \left(-\frac{U_{i j}}{k T}\right)\right) r^{2} d r \approx \frac{2 \pi}{3} r_{i j}^{3}$.

Finally, from Eqs. [A.3]- [A.6] one derives

$$
b=\sum_{i, j} x_{i} x_{j} b_{i j}, \quad c=\sum_{i, j} x_{i} x_{j} c_{i j}
$$

where $x_{i}=N_{i} / N$ are molar fractions.

In the case of ionic solution, $N_{i}$ is number of ions of the $i$ th species, and $N$ stands for the total number of ions.

For the sake of simplicity below we assume that all molecules have approximately the same size, that is, $b_{i j} \approx b$ for all $i$ and $j$. Then Eq. [A.7] yields

$$
b=\text { const. }
$$

In the system of hard spheres $(c=0)$ from Eqs. [A.1], [A.2], and [A.8] one derives

$$
\begin{aligned}
\mu_{i} & =\left(\frac{\partial F}{\partial N_{i}}\right)_{T, V, N_{k}} \\
& =\mu_{i}^{0}+k T\left[\ln \frac{n_{i}}{1-n b}+\frac{n b}{1-n b}\right],
\end{aligned}
$$

where $\mu_{i}$ is chemical potential, $\mu_{i}^{0}$ is standard chemical potential, and $n=N / V$. For low particle volume fraction one can write 


$$
\frac{n b}{1-n b} \approx \ln \left(1+\frac{n b}{1-n b}\right)=\ln \frac{1}{1-n b} \text {. }
$$

A combination of Eqs. [A.9] and [A.10] yields

$$
\mu_{i}=\mu_{i}^{0}+k T \ln \frac{n_{i}}{(1-n b)^{2}} \approx \mu_{i}^{0}+k T \ln \frac{n_{i}}{1-2 n b}
$$

Finally, setting equal the electrochemical potentials,

$$
\tilde{\mu}_{i}=\mu_{i}^{0}+k T \ln \frac{n_{i}}{1-2 n b}+Z_{i} e \psi+W_{i}
$$

of the ions in the bulk $(\psi \equiv 0)$ and in the double layer, one obtains Eq. [2.6] with $v=2 b$. In a first approximation the concentration of the coions in the double layer can be neglected. Then in accordance with Eq. [A.7] $v$ can be estimated as

$$
v \approx 2 b=2 \sum_{i, j} x_{i} x_{j} b_{i j}
$$

where $x_{i}$ is the fraction of the $i$ th counterion normalized with respect to the total number of counterions. The parameters $b_{i j}$ can be estimated from Eq. [A.6]. Thus for a single counterion in solution one has $x_{1}=1$ and $v$ is equal to 8 times the volume of the hard core.

\section{APPENDIX II}

\section{N umerical Method for Solving the Generalized Poisson- Boltzmann Problem}

Our purpose is to solve the equation

$$
\varphi^{\prime \prime}=f\left(x, \varphi, \varphi^{\prime}\right)
$$

where $f$ is a known function determined by Eqs. [2.2], [2.4], [2.6], [2.9], and [2.13]. We will utilize a partial linearization of Eq. [B.1] by means of the Newton method. Let $w(x)$ be a known trial function for the solution $\varphi(x)$. Then we expand the right-hand side of Eq. [B.1] in series for $\varphi \rightarrow w$

$$
\varphi^{\prime \prime}=f\left(x, w, w^{\prime}\right)+(\varphi-w) f_{\varphi}^{\prime}+\cdots
$$

where $\left.f_{\varphi}^{\prime} \equiv(\partial f / \partial \varphi)\right|_{\varphi=w}$. Next, we express $\varphi^{\prime \prime}$ by means of central differences

$$
\varphi^{\prime \prime}=\left(\varphi_{n+1}-2 \varphi_{n}+\varphi_{n-1}\right) / \Delta x^{2}+\mathrm{O}\left(\Delta x^{2}\right)
$$

where $\varphi_{n}=\left(x_{n}\right), x_{n}=x_{1}+(n-1) \Delta x, n=1,2,3, \ldots$, $N ; x_{N}=1 ; x_{1}=0$ for double layer or $x_{1}=x_{m}$ for thin film, see Fig. 1. Combining Eqs. [B.2] and [B.3] we obtain the following expression in terms of finite differences

$$
\begin{aligned}
a_{n} \varphi_{n-1}+b_{n} \varphi_{n}+c_{n} \varphi_{n+1}= & d_{n}, \\
& (n=2, \ldots, N-1),
\end{aligned}
$$

where

$$
\begin{aligned}
& a_{n}=c_{n}=1, \quad b_{n}=-\left(2+f_{\varphi}^{\prime} \Delta x^{2}\right), \\
& d_{n}=\left[f-w_{n} f_{\varphi}^{\prime}\right] \Delta x^{2} ; \quad w_{n} \equiv w\left(x_{n}\right) .
\end{aligned}
$$

We include three options for the three boundary conditions at the charged wall. In regime of fixed surface potential from Eq. [2.16] we obtain

$$
a_{N}=c_{N}=0, b_{N}=1, d_{N}=\varphi_{\mathrm{s}}(\text { fixed potential })
$$

The cases of fixed charge and charge regulation can be treated in a similar manner. Both Eqs. [2.17] and [2.18] may be expressed in the form

$$
\varphi_{\mathrm{s}}^{\prime}=g\left(\varphi_{\mathrm{s}}, \varphi_{\mathrm{s}}^{\prime}\right) ;\left.\quad \varphi_{\mathrm{s}}^{\prime} \equiv \varphi_{\mathrm{s}}^{\prime}\right|_{x=1}
$$

where $g\left(\varphi_{\mathrm{s}}, \varphi_{\mathrm{s}}^{\prime}\right)$ is a known function. By using the linearization

$$
\varphi_{\mathrm{s}}^{\prime}=g\left(w_{\mathrm{s}}, w_{\mathrm{s}}^{\prime}\right)+\left(\varphi_{\mathrm{s}}-w_{\mathrm{s}}\right) g_{\varphi_{\mathrm{s}}}^{\prime}+\cdots
$$

in terms of finite differences we get

$$
a_{N} \varphi_{N-1}+b_{N} \varphi_{N}=d_{N}
$$

where

$$
\begin{gathered}
a_{N}=2, \quad b_{N}=-\left(2+f_{\varphi}^{\prime} \Delta x^{2}\right)+2 g_{\varphi_{\mathrm{s}}}^{\prime} \Delta x, \\
d_{N}=\left(f-w_{N} f_{\varphi}^{\prime}\right) \Delta x^{2}-2\left(g-w_{N} g_{\varphi_{\mathrm{s}}}^{\prime}\right) \Delta x .
\end{gathered}
$$

In the case of fixed surface charge from Eq. [2.7] one derives

$$
g=\frac{4 \pi e \sigma_{\mathrm{s}}}{\kappa \varepsilon\left(w_{\mathrm{s}}^{\prime}\right) k T}, \quad g_{\varphi_{\mathrm{s}}}^{\prime}=0
$$

In the case of charge regulation from Eq. [2.18] one likewise obtains 


$$
\begin{gathered}
g\left(w_{\mathrm{s}}, w_{\mathrm{s}}^{\prime}\right) \\
=-\frac{4 \pi e^{2} \Gamma_{\mathrm{s}}}{\kappa \varepsilon\left(w_{\mathrm{s}}^{\prime}\right) k T\left(n_{1 \mathrm{~s}} A_{1}+1\right)}, A_{1} \equiv \frac{\delta_{1}}{\Gamma_{\mathrm{s}}} \exp \frac{\Phi}{k T}, \\
g_{\varphi_{\mathrm{s}}}^{\prime}=\frac{4 \pi e^{2} \Gamma_{\mathrm{s}} A_{1}}{\kappa \varepsilon\left(w_{\mathrm{s}}^{\prime}\right) k T\left(n_{1 \mathrm{~s}} A_{1}+1\right)^{2}}\left(\frac{\partial n_{1 \mathrm{~s}}}{\partial \varphi_{\mathrm{s}}}\right),
\end{gathered}
$$

where

$$
\begin{gathered}
\frac{\partial n_{1 \mathrm{~s}}}{\partial \varphi_{\mathrm{s}}}=n_{1 \mathrm{~s}}\left[v \sum_{k} Z_{k} n_{k \mathrm{~s}}-Z_{1}\left(1+v \sum_{k} n_{k \mathrm{~s}}\right)\right] /\left(1+v \sum_{k} n_{k \mathrm{~s}}\right) \\
n_{k \mathrm{~s}}=n_{k 0} e^{U_{k \mathrm{~s}}}, \quad U_{k \mathrm{~s}}=-Z_{k} w_{\mathrm{s}}-W_{k \mathrm{~s}} / k T
\end{gathered}
$$

From Eq. [2.15] one derives

$$
\begin{gathered}
b_{1} \varphi_{1}+c_{1} \varphi_{2}=d_{1}, \\
c_{1}=2, \quad b_{1}=-\left(2+f_{\varphi}^{\prime} \Delta x^{2}\right), \\
d_{1}=\left(f-w_{1} f_{\varphi}^{\prime}\right) \Delta x^{2} .
\end{gathered}
$$

The numerical procedure reduces to the iterative solution of the tridiagonal system of linear algebraic equations:

$$
\left(\begin{array}{cccccccc}
b_{1} & c_{1} & \cdot & \cdot & \cdot & \cdot & \cdot & d_{1} \\
a_{2} & b_{2} & c_{2} & \cdot & \cdot & (0) & \cdot & d_{2} \\
& & & & & & & \cdot \\
\cdot & \cdot & \cdot & \cdot & \cdot & \cdot & \cdot & \cdot \\
\cdot & (0) & \cdot & a_{n} & b_{n} & c_{n} & \cdot & d_{n} \\
\cdot & \cdot & \cdot & \cdot & \cdot & \cdot & \cdot & \cdot \\
& & & & & a_{N} & b_{N} & d_{N}
\end{array}\right) .
$$

For that purpose we used the standard Thomas method (33). Thus starting with $\left\{w_{n}\right\}_{n=1}^{N}$ as an initial approximation, we determine $\left\{\varphi_{n}\right\}_{n=1}^{N}$, which serves as the next approximation. Usually five to six iterations give sufficient accuracy since the Newton method is quadratically convergent.

We established that the above numerical scheme is considerably faster than that proposed by Gur et al. (19). After the solution, $\left\{x_{i}, \varphi_{i}\right\}_{i=1}^{N}$, is obtained, it and its derivative, $\left\{x_{i}, \varphi_{i}^{\prime}\right\}_{i=1}^{N}$, are interpolated by means of cubic splines, which are further substituted in the expressions containing $\varphi(x)$ and $\varphi^{\prime}(x)$.

\section{ACKNOWLEDGMENTS}

This study was supported financially by Colgate-Palmolive Inc., and in part, by the Bulgarian National Science Fund.

\section{REFERENCES}

1. Israelachvili, J. N., and Wennerström, H., J. Phys. Chem. 96, 520 (1992).

2. Israelachvili, J. N., and Adams, G. E., J. Chem. Soc. Faraday Trans. 1 74, 975 (1978).

3. Israelachvili, J. N., and Pashley, R. M., Nature 300, 341 (1982).

4. Pashley, R. M., J. Colloid Interface Sci. 80, 153 (1981).

5. Pashley, R. M., J. Colloid Interface Sci. 83, 531 (1981).

6. Israelachvili, J. N., "Intermolecular and Surface Forces." Academic Press, London, 1992.

7. Healy, T. W., Homolam, A., James, R. O., and Hunter, R. J., Faraday Discuss. Chem. Soc. 65, 156 (1978).

8. Marčelja, S., and Radič, N., Chem. Phys. Lett. 42, 129 (1976).

9. Schibi, D., and Ruckenstein, E., Chem. Phys. Lett. 95, 435 (1983).

10. Attard, P., and Batchelor, M. T., Chem. Phys. Lett. 149, 206 (1988).

11. Jönsson, B., and Wennerström, H., J. Chem. Soc. Faraday Trans. 2 79, 19 (1983).

12. Leikin, S., and Kornyshev, A. A., J. Chem. Phys. 92, 6890 (1990).

13. Henderson, D., and Lozada-Cassou, M., J. Colloid Interface Sci. 114, 180 (1986).

14. Henderson, D., and Lozada-Cassou, M., J. Colloid Interface Sci. 162, 508 (1994).

15. Henderson, D., in " Ordering and Organisation in Ionic Solutions" (N. Ise and I. Sogami, Eds.). World Scientific, Singapore, 1988.

16. Basu, S., and Sharma, M. M., J. Colloid Interface Sci. 165, 355 (1994).

17. Horn, R. G., Smith, D. T., and Haller, W., Chem. Phys. Lett. 162, 404 (1989).

18. Claesson, P., Carmona-Ribeiro, A. M., and Kurihara, K., J. Phys. Chem. 93, 917 (1989).

19. Gur, Y., Ravina, I., and Babchin, A. J., J. Colloid Interface Sci. 64, 333 (1978).

20. Booth, F., J. Chem. Phys. 19, 391 (1951).

21. Born, M., Z. Phys. 1, 45 (1920).

22. Bikerman, J. J., Philos. Mag. 33, 384 (1942).

23. Rowlinson, J. S., in "Fundamentals of Inhomogeneous Fluids" (D. Henderson, Ed.). Dekker, New York, 1992.

24. Shchukin, E. D., Pertsov, A. V., and Amelina, E. A., "Colloid Chemistry.,' Moscow Univ. Press, Moscow, 1982. [In Russian]

25. Verwey, E. J. W., and Overbeek, J. Th. G., "The Theory of Stability of Liophobic Colloids.' Elsevier, Amsterdam, 1948.

26. Derjaguin, B. V., Churaev, N. V., and Muller, V. M., "Surface Forces." Plenum Press, Consultant's Bureau, New York, 1987.

27. Langmuir, I., J. Chem. Phys. 6, 873 (1938).

28. Kralchevsky, P. A., and Paunov, V. N., Colloids Surfaces 64, 245 (1992).

29. Kralchevsky, P. A., and Denkov, N. D., Chem. Phys. Lett. 240, 385 (1995).

30. Miklavic, S. J., and Ninham, B. W., J. Colloid Interface Sci. 134, 305 (1990).

31. Hill, T. L., “An Introduction to Statistical Thermodynamics.' Addison-Wesley, Reading, MA, 1962.

32. Landau, L. D., and Lifshitz, E. M., 'Statistical Physics,'” Part 1. Pergamon Press, Oxford, 1980.

33. Press, W. H., Teukolsky, S. A., Vetterling, W. T., and Flannery, B. P., "Numerical Recipes in FORTRAN,", second ed. Cambridge Univ. Press, Cambridge, 1992. 